\title{
Prenatally Diagnosed Rare Trisomy 16 Mosaicism in Human Amniotic Fluid Cells in the Second Trimester: A Case Report
}

\author{
Sook Ryung Kim¹, Eun Jung Choi ${ }^{1}$, Young Joo Kim¹, Tae Yoon Kim², and ${ }^{\dagger}{ }^{1}$ oung Jin Lee ${ }^{1}$ \\ ${ }^{1}$ iDream Research Center, Mizmedi Hospital, Seoul 07639, Korea \\ ${ }^{2}$ Dept. of Obstetrics and Gynecology, Mizmedi Hospital, Seoul 07639, Korea
}

\begin{abstract}
Although trisomy 16 is commonly detected in spontaneous abortions and accounts for over $30 \%$ of cases of autosomal trisomy detected after spontaneous abortion, trisomy 16 mosaicism is rarely detected by amniocentesis in the second trimester. Here, we report a case of level III trisomy 16 mosaicism $(47, X X,+16[8] / 46, X X[31])$ diagnosed by cytogenetic analysis of independently cultured amniotic fluid cells. The female baby was delivered at full term with low birth weight and intrauterine growth retardation, and interestingly, her karyotype was normal (46,XX). Given the difficulty in predicting the outcomes of fetuses with this mosaicism, it is recommended to inform the possibility of mosaicisms including this trisomy 16 mosaicism during prenatal genetic diagnosis and genetic counseling for parents.
\end{abstract}

Key words : Prenatal cytogenetic diagnosis, Trisomy 16, Mosaicism, Amniocentesis

\section{INTRODUCTION}

Trisomy 16 accounts for $1-2 \%$ or more of all first trimester miscarriages (Neiswanger et al., 2006), and the abortuses show empty sacs, disorganized embryos, or minimal embryonic development (Benn, 1998). Trisomy 16 occurs almost exclusively as a non-disjunction event in maternal meiosis I, while gestational trisomy 16 mosaicism is caused by mitotic non-disjunction (Neiswanger et al., 2006). Trisomy 16 mosaicism develops through the postzygotic loss of one copy of chromosome 16 , which rescues in parts of the trisomic embryo and/or placenta, and maternal uniparental disomy (UPD) is noted in approximately a third of such mosaicism cases. It is rarely observed in amniotic fluid cells, and is associated with a high risk of abnormal outcomes such as intrauterine growth retardation (IUGR), fetal death in utero, preeclampsia, preterm delivery, neonatal death, developmental delay, congenital heart defects, and other minor anomalies (Chareonsirisuthigul et al., 2014).

Although trisomy 16 accounts for over $30 \%$ of cases of autosomal trisomy detected after spontaneous abortion, the detection of trisomy 16 mosaicisms by amniocentesis in the second trimester is rare (Neiswanger et al., 2006). Moreover, there remains a high probability of gestational abnormalities that result from trisomy 16 mosaicism. However, prenatal mosaic trisomy 16 cells are difficult to observe in fetal lymphocytes and are rarely confirmed in postnatal blood or other tissues (Benn, 1998).

Here, we report a rare case of level III trisomy 16 mosai-

Manuscript received April 30, 2018, Received in revised form May 1, 2018, Accepted June 12, 2018

${ }^{\dagger}$ Corresponding Author : Young Jin Lee, iDream Research Center, Mizmedi Hospital, Seoul 07639, Korea. Tel: +82-2-2007-1840, Fax: +82-2-2007-1852, E-mail: youngjin.lee@mizmedi.com

This is an Open Access article distributed under the terms of the Creative Commons Attribution Non-Commercial License (http:// creative-commons.org/licenses/by-nc/3.0) which permits unrestricted non-commercial use, distribution, and reproduction in any medium, provided the original work is properly cited. 
cism $(47, \mathrm{XX},+16[8] / 46, \mathrm{XX}[31])$ diagnosed by cytogenetic analysis of independently cultured amniotic fluid cells during the second trimester.

\section{CASE}

A 32-year-old female, with no remarkable medical history and one prior pregnancy, was referred for amniocentesis after positive maternal serum marker screening at $18+6$ weeks of gestation. The chromosomal status of amniotic fluid cells at 18 weeks of gestation and postnatal peripheral blood were analyzed using conventional cytogenetic methods per the International System for Human Cytogenetic Nomenclature 2013 guidelines (Shaffer et al., 2013).

The fetal karyotype was 47,XX+16[8]/46,XX[31] (20.5\% mosaicism) in independent cultured cells (Fig. 1A \& B), and cells with trisomy 16 were detected in all culture dishes (level III). Due to the medical condition (delayed amniocentesis with small amount of amniotic fluid) of the mother and the fetus, we were unable to perform repeated amniocentesis and interphase fluorescence in situ hybridization to validate the previous results.

Many published cases of mosaic trisomy 16 were identified as serum test positive (abnormal) using blood samples from the mothers (Neiswanger et al., 2006; Hidaka et al., 2009). In our case, elevated maternal serum alpha fetoprotein (MSAFP) levels $(93.6 \mathrm{IU} / \mathrm{mL})$ and abnormal triple test results (human chorionic gonadotropin (hCG) $647 \mathrm{IU} / \mathrm{mL}$, unconjugated estriol (uE3) $0.8 \mathrm{ng} / \mathrm{mL}$, and inhibin-A 473.9 $\mathrm{pg} / \mathrm{mL}$ ) were identified, which may reflect an association between low uE3 and growth retardation (Benn, 1998).

No abnormalities except a dacryocystocele were observed by ultrasonography, and the female baby was born by normal spontaneous vaginal delivery at full term with low birth weight $\left(2,250 \mathrm{~g}, 3^{\text {rd }}\right.$ of $100^{\text {th }}$; Standard growth chart, The Korea Center for Disease Control and Prevention). Based on previous report (Astner et al., 1998) involved in the detection of fetal and placental abnormalities
(A)

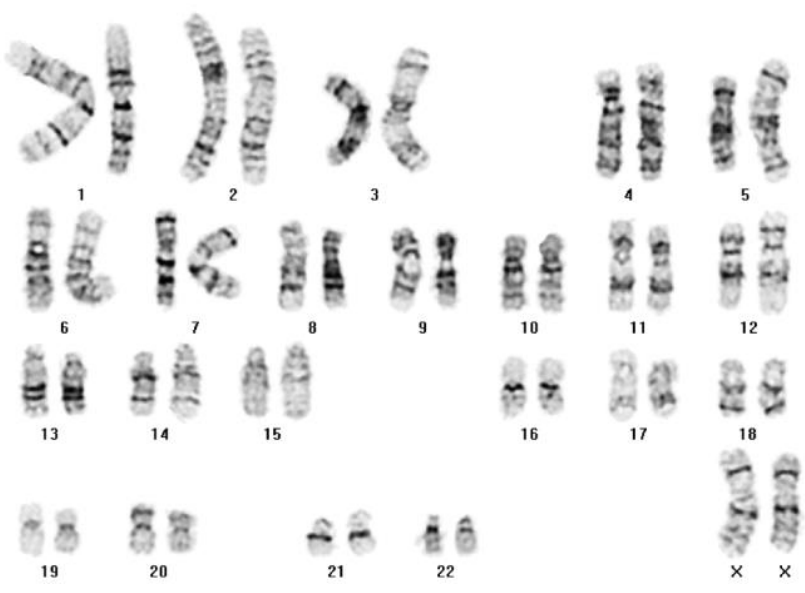

(B)

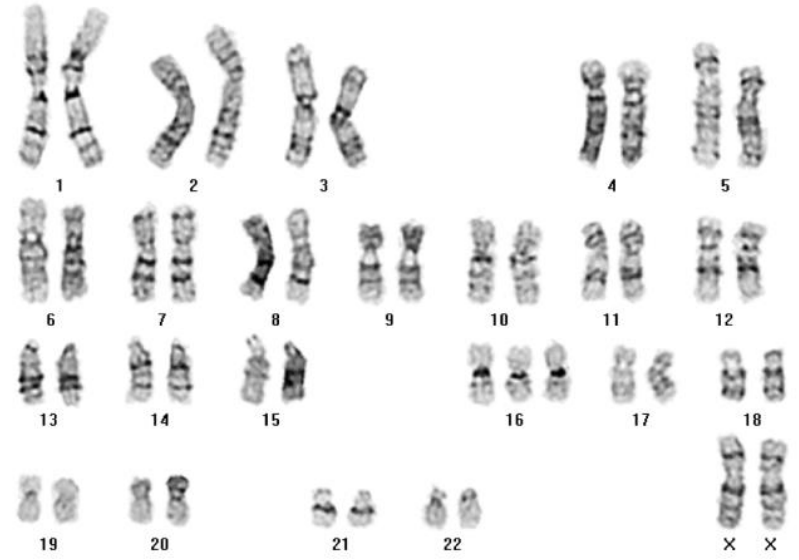

Fig. 1. Prenatal chromosomal analysis of amniotic fluid cells. (A) 46,XX (B) 47,XX,+16.

associated with trisomy 16 by sonography, brain sonography was performed and showed no evidence of abnormal increased echoes in the brain parenchyma or ventricles, with normal posterior fossa, breathing, and heart sounds. A normal karyotype $(46, \mathrm{XX})$ by 100 phases analysis was observed in the peripheral blood of the newborn (Fig. 2), which was fed well and displayed unremarkable breathing, heart sounds, and umbilicus and oral cavity findings.

\section{DISCUSSION}

Mosaicism refers to the presence of cells with two or more different genotypes in an individual or tissue sample 


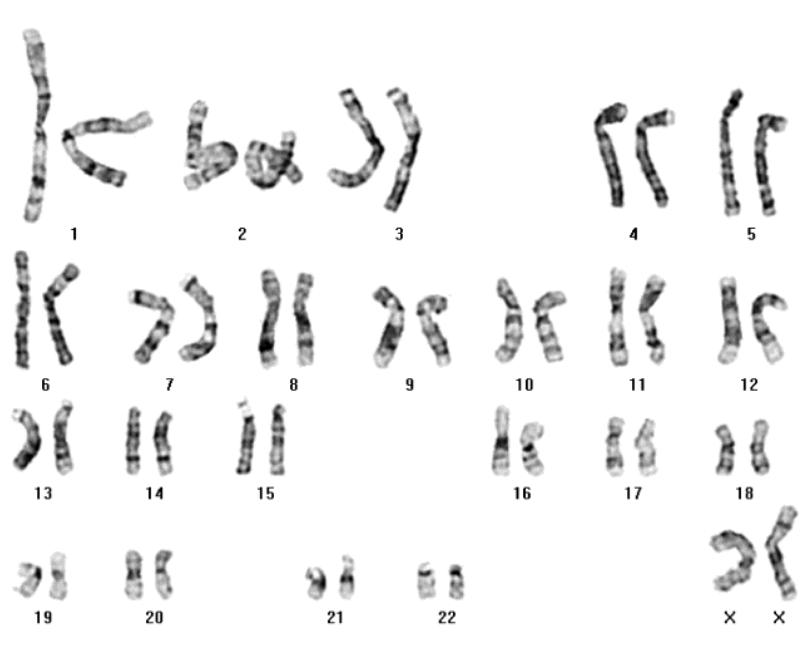

Fig. 2. Postnatal chromosomal analysis of peripheral blood, showing a 46,XX karyotype.

(Haddad et al., 2013). A mitotic error in epiblast may produce mosaicism of both embryonic and amniotic tissue. Of these, separating confined placental mosaicism and pseudo-mosaicism from true mosaicism is critical. This based on the number of abnormal cells seen and whether one or more than one presumptive abnormal clone exists. Mosaicisms are classified as level I to III depending on the amount of abnormal cells (Mascarello et al., 2011), and level I mosaicism is seen $2.3-7 \%$, level II in $0.7-1.1 \%$, and level III in about $0.2 \%$ of amniocentesis (Wilson et al., 1989). Our case was identified as level III mosaicism and we conclude this mosaicism is true.

When trisomy mosaicism is observed in cultured fetal cells during prenatal diagnosis, difficulties can occur in interpreting whether the fetus is truly mosaic and in determining the clinical significance of the apparent mosaicism. Although true mosaicism is usually associated with a high risk of mosaicism in the fetus (Haddad et al., 2013), it should be carefully considered during prenatal diagnosis, as various degrees of mosaicism and specimen types can be observed depending on the gestational age.

The presence of trisomy 16 in amniocentesis is concerning, because it can result in low birth weight and a high risk of malformation (Langlois et al., 2006). Trisomy 16 is also associated with IUGR, orofacial clefting, cardiac defects, renal dysplasia, imperforate anus, and many other anomalies (Yong et al., 2003; Sparks et al., 2017), but the lack of published data on the long-term outcome of these children makes it difficult to counsel parents after a prenatal diagnosis of mosaicism for trisomy 16 (Langlois et al., 2006). The level of trisomy observed by amniocentesis is influenced by stochastic processes during cell proliferation, differentiation, and development (Yong et al., 2003); however, it is unclear whether low levels of trisomic cells are necessarily harmful or result in an abnormal phenotype.

Non-invasive prenatal genetic testing, also known as cell-free fetal DNA testing, has been used since 2011 to screen for common fetal aneuploidies, and is highly sensitive and specific, with a pooled sensitivity and specificity of 97.4 and $99.3 \%$, respectively, for trisomy 13,18 , and 21 (Mardy and Wapner, 2016). Since low-level true fetal mosaicism is possible even with a negative amniotic fluid culture, patients should be informed of this possibility (Grati et al., 2014). The identification of trisomy mosaicism during prenatal diagnosis is often shrouded in uncertainty for the genetic counselor, and more importantly, for the parents. In up to $10 \%$ of all trisomy 16 pregnancies, postzygotic rescue may occur, enabling the pregnancy to continue with the usual complement of two copies of chromosome 16 (Sparks et al., 2017). However, the risk of residual mosaicism exists with trisomic rescue, and limited information is available about the outcomes of pregnancies with trisomy 16 mosaicism (Sparks et al., 2017); this might be considered normal, but abnormalities and even in utero death could occur depending on the chromosomal status and the degree of mosaicism (Coman et al., 2010). Regarding to verify rare mosaicism after amniocentesis, cordocentesis is another option that helps to counsel the parents about the prognosis (Post and Nijhuis, 1992; Schwartz \& Raffel, 1992).

Although studies of affected children and evaluation of important long-term outcomes remain to be conducted, 
there are several limitations to consider. Recently, a study found that the majority of children with trisomy 16 mosaicism and confined placental mosaicism during pregnancy or at birth exhibited normal neurodevelopmental outcomes and high quality of life scores (Generic Core Scales were used to measure health-related quality of life, as well as physical, emotional, social, and educational performance) (Sparks et al., 2017). However, the role of UPD remains unclear, because this testing was only performed for eight cases in the cohort. Another study reported that a baby with trisomy 16 mosaicism had normal development, with postnatal growth retardation reversal (Coman et al., 2010). Therefore, parental counseling is prudent after a diagnosis of trisomy 16 mosaicism by amniocentesis.

Taken together, these results suggest that given the difficulty in predicting its outcomes, rare trisomy 16 mosaicism should be considered during prenatal genetic diagnosis and parental genetic counseling.

\section{ACKNOWLEDGEMENTS}

This study was supported by the Mizmedi Hospital Research Fund.

\section{REFERENCES}

Astner A, Schwinger E, Caliebe A, Jonat W, Gembruch U (1998). Sonographically detected fetal and placental abnormalities associated with trisomy 16 confined to the placenta. A case report and review of the literature. Prenat Diagn 18:1308-1315.

Benn P (1998). Trisomy 16 and trisomy 16 mosaicism: A review. Am J Med Genet 79:121-133.

Chareonsirisuthigul T, Worawichawong S, Parinayok R, Promsonthi P, Rerkamnuaychoke B (2014). Intrauterine growth retardation fetus with trisomy 16 mosaicism. Case Rep Genet 2014:739513.

Coman D, Gardner RJM, Pertile MD, Kannu P (2010). Tri- somy 16 mosaicism at chorionic villus sampling and amniocentesis with a normal physical and intellectual outcome. Fetal Diagn Ther 28:117-118.

Grati FR, Malvestiti F, Ferreira JC, Bajaj K, Gaetani E, Agrati C, Grimi B, Dulcetti F, Ruggeri AM, De Toffol S, Maggi F, Wapner R, Gross S, Simoni G (2014). Fetoplacental mosaicism: Potential implications for falsepositive and false-negative noninvasive prenatal screening results. Genet Med 16:620-624.

Haddad G, He W, Gill J, Witz C, Wang C, Kaskar K, Wang W (2013). Mosaic pregnancy after transfer of a "euploid" blastocyst screened by DNA microarray. J Ovarian Res 6:70.

Hidaka N, Yamamoto N, Tsukimori K, Hojo S, Suzuki SO, Wake N (2009). Prenatal diagnosis of trisomy 16 mosaicism manifested as pulmonary artery stenosis. J Clin Ultrasound 37:107-111.

Langlois S, Yong PJ, Yong SL, Barrett I, Kalousek DK, Miny P, Exeler R, Morris K, Robinson WP (2006). Postnatal follow-up of prenatally diagnosed trisomy 16 mosaicism. Prenat Diagn 26:548-558.

Mardy A, Wapner RJ (2016). Confined placental mosaicism and its impact on confirmation of NIPT results. Am J Med Genet C Semin Med Genet 172:118-122.

Mascarello JT, Hirsch B, Kearney HM, Ketterling RP, Olson SB, Quigley DI, Rao KW, Tepperberg JH, Tsuchiya KD, Wiktor AE (2011). Section E9 of the American College of Medical Genetics technical standards and guidelines: Fluorescence in situ hybridization. Genet Med 13:667-675.

Neiswanger K, Hohler PM, Hively-Thomas LB, McPherson EW, Hogge WA, Surti U (2006). Variable outcomes in mosaic trisomy 16: Five case reports and literature analysis. Prenat Diagn 26:454-461.

Post JG, Nijhuis JG (1992). Trisomy 16 confined to the placenta. Prenat Diagn 12:1001-1007.

Schwartz S, Raffel LJ (1992). Prenatal detection of 45,X/ 46,XX/47,XXX mosaicism through amniocentesis: mo- 
saicism confirmed in cord blood, amnion, and chorion. Prenat Diagn 12:1043-1046.

Shaffer LG, McGowan-Jordan J, Schmid M (2013). ISCN 2013: An International System for Human Cytogenetic Nomenclature (2013). Karger, Basel.

Sparks TN, Thao K, Norton ME (2017). Mosaic trisomy 16: What are the obstetric and long-term childhood outcomes? Genet Med 19:1164-1170.

Wilson MG, Lin MS, Fujimoto A, Herbert W, Kaplan FM (1989). Chromosome mosaicism in 6,000 amniocenteses. Am J Med Genet 32:506-513.

Yong PJ, Barrett IJ, Kalousek DK, Robinson WP (2003). Clinical aspects, prenatal diagnosis, and pathogenesis of trisomy 16 mosaicism. J Med Genet 40:175-182. 